\title{
(Anti)hypertriton lifetime puzzle
}

\author{
YU-GANG MA
}

\begin{abstract}
Shanghai Institute of Applied Physics, Chinese Academy of Sciences, Shanghai 201800, China
\end{abstract}

\begin{abstract}
Most calculations on the lifetime of (anti)hypertriton gave a similar lifetime which is close to the lifetime of free $\Lambda$ decays. However, recent measurements on (anti)hypertriton lifetime demonstrate a much short lifetime. All results for (anti)hypertriton lifetime by two-body decay channel of ${ }^{3} \mathrm{He}+\pi$ for $\mathrm{Au}+\mathrm{Au}$ collision at $\mathrm{RHIC}, \mathrm{Pb}+\mathrm{Pb}$ collision at $\mathrm{LHC}$ and $\mathrm{Li}+\mathrm{C}$ collisions at GSI show a significant short lifetime in comparison with lifetime of free $\Lambda$ decays. However, theoretical interpretation remains puzzle.
\end{abstract}

Hypernucleus provides an ideal environment to learn the hyperonnucleon interaction, which is responsible partly for the binding of hypernuclei and lifetime. Therefore, a meaurement of the precise lifetime of hypernucleus could provide a tool to investigate of hyeron-nucleon interaction [1-7]. The most simple hypernucleus is hypertriton, which is a bound state of a proton, a neutron, and a hyperon $(\Lambda$ or $\Xi)$, it is bound with respect to the $\Lambda$ d threshold by $0.136 \pm 0.05 \mathrm{MeV}$. In $2010,3 \bar{\Lambda} \overline{\mathrm{H}}$, the antimatter partner of ${ }_{\Lambda}^{3} \mathrm{H}$, has been discovered at RHIC-STAR as the first antimatter hypernucleus [8] - The hypertriton decays weakly into mesonic and nonmesonic channels. The nonmesonic decay channels are ${ }_{\Lambda}^{3} \mathrm{H} \rightarrow \mathrm{d}+\mathrm{n}$ and ${ }_{\Lambda}^{3} \mathrm{H} \rightarrow \mathrm{p}+\mathrm{n}+\mathrm{n}$. In the mesonic decay mode there are more channels: ${ }_{\Lambda}^{3} \mathrm{H} \rightarrow \pi^{-}\left(\pi^{0}\right)+{ }^{3} \mathrm{He}\left({ }^{3} \mathrm{H}\right)$, ${ }_{\Lambda}^{3} \mathrm{H} \rightarrow \pi\left(\pi^{0}\right)+\mathrm{d}+\mathrm{p}(\mathrm{n})$, and ${ }_{\Lambda}^{3} \mathrm{H} \rightarrow \pi\left(\pi^{-}\right)+\mathrm{p}+\mathrm{n}+\mathrm{p}(\mathrm{n})$. In contrast to heavier hypernuclei, where mesonic decays are Pauli blocked, here in the hypertriton they are by far the dominant ones. Further there are experimental data on the branching ratio $R=\Gamma\left({ }_{\Lambda}^{3} \mathrm{H} \rightarrow \pi^{-}+{ }^{3} \mathrm{He}\right) / \Gamma\left({ }_{\Lambda}^{3} \mathrm{H} \rightarrow\right.$ all $\pi^{-}$meson modes) ranging between $0.30 \pm 0.07[9]$ and $0.39 \pm 0.07$ [10]. From the 
technique viewpoint in heavy-ion collision, a simple identification method of ${ }_{\Lambda}^{3} \mathrm{H}\left({ }_{\Lambda}^{3} \overline{\mathrm{H}}\right)$ can be achieved by reconstructing their secondary vertex via the decay channel of ${ }_{\Lambda}^{3} \mathrm{H} \rightarrow{ }^{3} \mathrm{He}+\pi^{-}\left({ }_{\Lambda}^{3} \overline{\mathrm{H}} \rightarrow{ }^{3} \overline{\mathrm{He}}+\pi^{+}\right)$, which occurs with a branching ratio of $25 \%$ (assuming that this branching fraction is the same as that for ${ }_{\Lambda}^{3} \mathrm{H}$ [2] $)[8,11,12]$. In contrast, reconstruction of ${ }_{\Lambda}^{3} \mathrm{H}\left({ }_{\Lambda}^{3} \overline{\mathrm{H}}\right)$ from three-body decay, i.e. ${ }_{\Lambda}^{3} \mathrm{H} \rightarrow \pi\left(\pi^{0}\right)+\mathrm{d}+\mathrm{p}(\mathrm{n})$, is more complicate, but it deserves to check with two-body decay results, and it is under progress well [13].

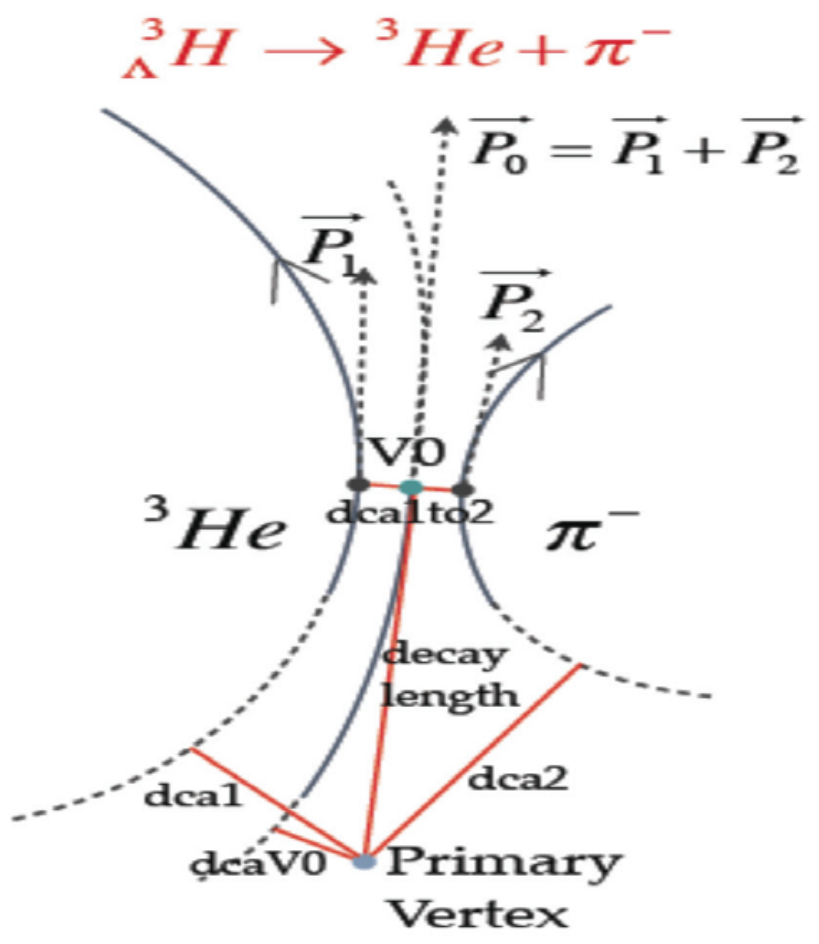

Figure 1: Topological decay map for 2-body channel of ${ }_{\Lambda}^{3} \mathrm{H}$.

Theoretically, most of calculations predicted that the hypertriton lifetime is a littler shorter than the free $\Lambda$ lifetime since it could exhibit the modification of the $\Lambda$ wavefunction in the nuclear medium. For an example, the $\pi$-mesonic decay of the hypertriton is calculated based on a hypertriton wave function and $3 \mathrm{~N}$ scattering states, which are solutions of three-body Faddeev equations using realistic NN and hyperon-nucleon interactions [14]. They predicted that the total lifetime of ${ }_{\Lambda}^{3} \mathrm{H}$ is 256 ps which is $3 \%$ smaller than for the free $\Lambda$ particle [14]. 
In this work, the lifetime measurement data for ${ }_{\Lambda}^{3} \mathrm{H} \quad\left({ }_{\Lambda}^{3} \overline{\mathrm{H}}\right)$ two-body decay from $\mathrm{Au}+\mathrm{Au}$ collisions at RHIC is reported. The data was collected by the STAR experiment at RHIC, using the cylindrical TPC, which is 4 meters in diameter and 4.2 meters long in the beamline direction [15]. The identification of tracks can be achieved by correlating their ionization energy loss $\langle d E / d x\rangle$ in TPC with their magnetic rigidity.

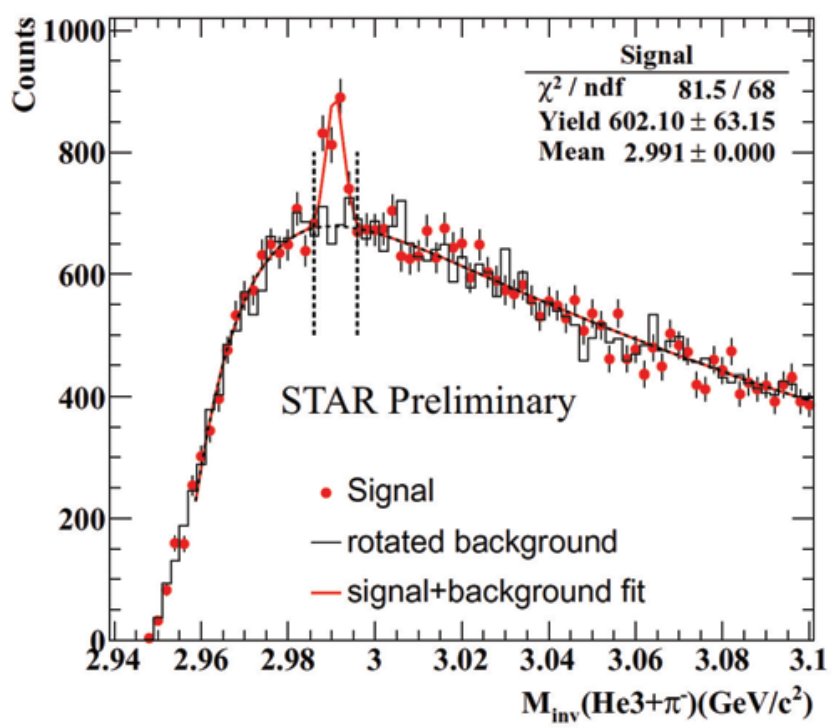

Figure 2: Reconstructed invariant mass distribution of ${ }_{\Lambda}^{3} \mathrm{H}$ from two-body decay channel of ${ }^{3} \mathrm{He}$. Solid circles stand for the signal distribution, while dashed lines are the rotated combinatorial background. Vertical dashed lines represent the mass range we use for bin counting of ${ }_{\Lambda}^{3}$ Hyield. Adapted from Ref. [12].

For two-body decay channel, topological cuts include the distance between two daughter tracks ${ }^{3} \mathrm{He}\left({ }^{3} \overline{\mathrm{He}}\right)$ and $\pi^{-}\left(\pi^{+}\right)(<1 \mathrm{~cm})$, distance of closest approach $(\mathrm{DCA})$ between ${ }_{\Lambda}^{3} \mathrm{H} \quad\left({ }_{\Lambda}^{3} \overline{\mathrm{H}}\right)$ and primary vertex $(<1 \mathrm{~cm})$, decay length of ${ }_{\Lambda}^{3} \mathrm{H} \quad\left({ }_{\bar{\Lambda}}^{3} \overline{\mathrm{H}}\right)(>2.4 \mathrm{~cm})$, and the DCA of $\pi$ track $(>0.8 \mathrm{~cm})$, are employed to enhance the signal to background ratio [12]. Figure 1 shows the topological decay maps for both 2-body channel. The invariant mass of ${ }_{\Lambda}^{3} \mathrm{H} \quad\left({ }_{\Lambda}^{3} \overline{\mathrm{H}}\right)$ was calculated based on the conservation of momentum and energy in the decay process. The result is shown in Figure 2. The successfully reproduced combinatorial background with a rotation strategy can be described by double exponential function: $f(x) \propto \exp \left[-\left(x / p_{1}\right)\right]-\exp \left[-\left(x / p_{2}\right)\right]$, where $x=m-m\left({ }^{3} \mathrm{He}\right)-m(\pi)$, and $p_{1}, p_{2}$ are the free parameters. Finally, the signals are counted by subtracting the double exponential background 
of ${ }_{\Lambda}^{3} \mathrm{H}$ and ${ }_{\bar{\Lambda}}^{3} \overline{\mathrm{H}}$.

To demonstrate a visual scenario for ${ }_{\Lambda}^{3} \mathrm{H}$ decay, a carton picture is depicted to help us understand the possible decay mechanism. Figure 3 shows a possible decay process of two-body decay of ${ }_{\Lambda}^{3} \mathrm{H}$, which can be understood by a capture of decayed proton from $\Lambda$ by the existed neutron and proton inside ${ }_{\Lambda}^{3} \mathrm{H}$, resulting in a final decayed products ${ }^{3} \mathrm{He}$ and $\pi$.

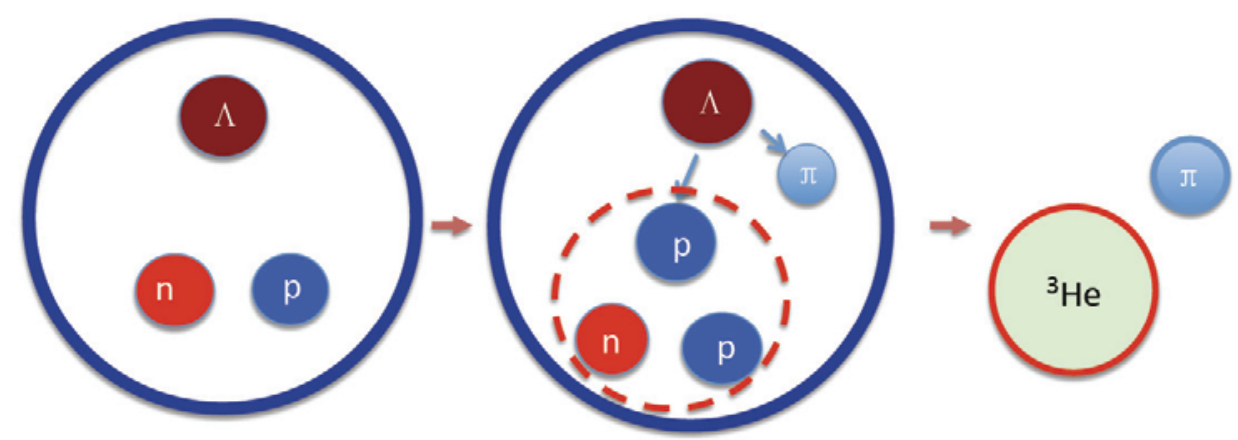

Figure 3: Cartons for ${ }_{\Lambda}^{3} \mathrm{H}\left({ }_{\Lambda}^{3} \overline{\mathrm{H}}\right)$ two-body decay channel of ${ }^{3} \mathrm{He}+\pi$.

The above secondary vertex reconstruction of ${ }_{\Lambda}^{3} \mathrm{H}\left({ }_{\Lambda}^{3} \overline{\mathrm{H}}\right)$ allows ones to perform a calculation of its lifetime, via equation $N(t)=N(0) \exp (-t / \tau)$, where $t=l /(\beta \gamma c), \beta \gamma c=p / m, l$ is the decay length of ${ }_{\Lambda}^{3} \mathrm{H}, p$ is their momentum, $m$ is their mass value, while $c$ is the speed of light. ${ }_{\Lambda}^{3} \mathrm{H}$ and ${ }_{\Lambda}^{3} \overline{\mathrm{H}}$ samples are combined together to get a better statistics, with the assumption of the same lifetime of ${ }_{\Lambda}^{3} \mathrm{H}$ and $\frac{3}{\bar{\Lambda}} \overline{\mathrm{H}}$ based on the CPT symmetry theory. The measured yield is corrected for the tracking efficiency and acceptance of TPC, as well as the reconstruction efficiency of ${ }_{\Lambda}^{3} \mathrm{H}$ and ${ }_{\Lambda}^{3} \overline{\mathrm{H}}$. Then, the $l /(\beta \gamma)$ distribution can be fitted with an exponential function to extract the lifetime parameter $c \tau$.

The RHIC beam energy scan program in 2010-2011 allowed STAR to collect data for $\mathrm{Au}+\mathrm{Au}$ collisions over a broad range of energies. To get an even better statistics, datasets are combined in the lifetime measurement. For two-body decay, the best fitting with $\chi^{2}$ minimization method gives a value of $c \tau=3.69 \pm_{0.65}^{0.77} \mathrm{~cm}$, which corresponds to a lifetime of $123 \pm{ }_{22}^{26}$ (stat) \pm 10 (sys) ps (Fig. 4) [12]. As a comparison, STAR $2010{ }_{\Lambda}^{3} \mathrm{H}$ lifetime measurement [8] and the STAR 2010+2012 combined results are also provided. The current measurement is consistent with the STAR 2010 measurement within $1.5 \sigma$ and is statistically improved. In this lifetime measurement, two kinds of sources for systematic study are considered: 1 . choice of V0 topol- 


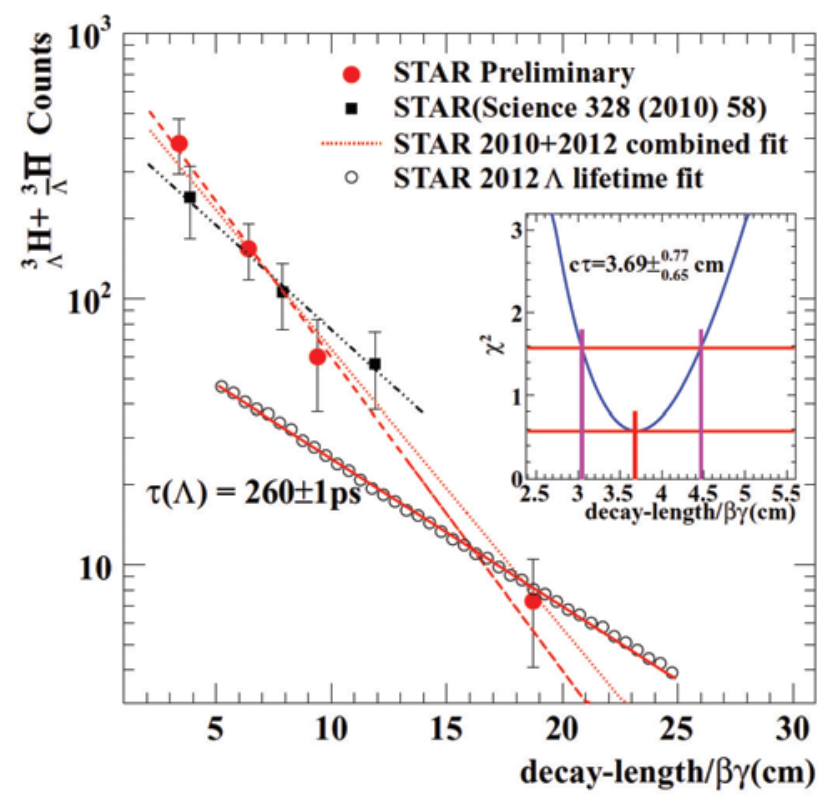

Figure 4: The yields of ${ }_{\Lambda}^{3} \mathrm{H}+{ }_{\bar{\Lambda}}^{3} \overline{\mathrm{H}}$ and $\Lambda$ (open circles) vs $c \tau$ distribution. The solid lines stand for the $c \tau$ fits, and the insert plot describes $\chi^{2}$ distribution of the best fits. Comparison between the present measurement and the previous measurements is also demonstrated. Adapted from Ref. [12].

ogy cuts; 2. choice of bin width and invariant mass range. These effects contribute to the final systematic error. Additional sources of loss, like the interaction between ${ }_{\Lambda}^{3} \mathrm{H}$ and material (air+detector) are also considered, which can be neglected due to its less than $1.5 \%$ effect [12]. As a further cross-check, $\Lambda$ is reconstructed via the $\Lambda \rightarrow p+\pi^{-}$decay channel. To this end, the same method is adopted to obtain the $\Lambda$ lifetime and the result is $260 \pm 1$ ps which is consistent with the $\tau=263 \pm 2$ ps compiled by the Particle Data Group [16]. In this way, we confirm that the method to extract ${ }_{\Lambda}^{3} \mathrm{H}\left(\frac{3}{\Lambda} \overline{\mathrm{H}}\right)$ lifetime is reliable.

In summary, with the current highest statistics of (anti-)hypertriton samples, the results of STAR measurement definitely gives a short lifetime from two-body decay channel, $123_{-22}^{+26} p s$, which is much shorter than the free $\Lambda$ decay lifetime. Also, recent measurements of ALICE collaboration for $\mathrm{Pb}$ $+\mathrm{Pb}$ collisions at $\sqrt{s_{N N}}=2.76 \mathrm{TeV}$ show $\tau=181_{-39}^{+54}$ (stat.) \pm 33 (syst.) ps for ${ }_{\Lambda}^{3} \mathrm{H}$ lifetime [17] and HypHI Collaboration for ${ }^{6} \mathrm{Li}$ projectiles at $2 \mathrm{~A}$ $\mathrm{GeV}$ on a carbon target also gave $\tau=183_{-32}^{+42} \pm 37$ ps for ${ }_{\Lambda}^{3} \mathrm{H}$ lifetime [18]. All these results are consistent within the errors and demonstrated that 
${ }_{\Lambda}^{3} \mathrm{H}\left({ }_{\Lambda}^{3} \overline{\mathrm{H}}\right)$ decay has a significant short lifetime than theoretical expected values. Therefore an open question remains for theoretical interpretation, which still leave us a puzzle for understanding the structure of ${ }_{\Lambda}^{3} \mathrm{H}\left({ }_{\Lambda}^{3} \overline{\mathrm{H}}\right)$ and hyperon-nucleon interaction.

This work is partially supported by the 973 program under contract no. 2014CB845401 and NSFC under contract Nos. 11421505, 11035009 and 11220101005 .

\section{References}

[1] R. H. Dalitz, G. Rajasekharan, Phys. Lett. 1, 58 (1962)

[2] H. Kamada, J. Golak, K. Miyagawa, H. Witala, W. Glockle, Phys. Rev. C 57, 1595 (1998)

[3] B. F. Gibson, E.V. Hungerford III, Phys. Rep. 257, 349 ( 1995)

[4] E. Oset, P. Fernandez de Cordoba, L.L. Salcedo, R. Brockmann, Phys. Rep. 188, 79 (1990)

[5] E. Hiyama, T. Motoba, T. A. Rijken and Y. Yamamoto, Progress of Theoretical Physics Supplement 185, 1 (2010)

[6] A. S. Botvinaa, J. Steinheimer, E. Bratkovskaya, M. Bleicher, J. Pochodzalla, Phys. Lett. B 742, 7 (2015)

[7] H. Shen, J. S. Liang, Nuclear Techniques (in Chinese) 37 , 100507 (2014)

[8] B. I. Abelev et al. (STAR Collaboration), Science 328 , 58 (2010)

[9] G. Keyes et al., Phys. Rev. D 1, 66 (1970); Phys. Rev. Lett. 20, 819 (1968); Nucl. Phys. B 67, 269 (1973)

[10] M. Block et al., in Proceedings of the Sienna International Conference on Elementary Particles, edited by G. Bernandini and G. P. Puppi Societa de Fisica, Bologna, 1963, p. 62.

[11] J. H. Chen, Nucl. Phys. A 835, 117 (2010); Nucl. Phys. A 830 , 761c (2009)

[12] Y. H. Zhu, Nucl. Phys. A 904-905, 551c (2013); PhD Thesis (2013), Shanghai Institute of Applied Physics, CAS, 2013. 
[13] Y. F. Xu for the STAR Collaboration, the talk at the 12th International Conference on Hypernuclear and Strange Particle Physics (HYP2015), Sept 2015, Sendai, Japan.

[14] H. Kamada, J. Golak, K. Miyagawa, H. Witala, and W. Glöckle, Phys. Rev. C 57, 1595 (1998)

[15] M. Anderson et al., Nucl. Instrum. Methods A 499, 659 (2003)

[16] C. Amsler et al., Phys. Lett. B 667, 1 (2008)

[17] J. Adam et al. (ALICE Collaboration), arXiv:1506.08453.

[18] C. Rappold, E. Kim, D. Nakajima, T. Saito, O. Bertini et al. (HypHI Collaboration), Nucl. Phys. A 913, 170 (2013) 